\title{
Genetic construction, expression, and characterization of a single chain anti-CEA antibody fused to cytosine deaminase from yeast
}

\author{
SILVIA ZAMBONI, ALESSANDRA MALLANO, MICHELA FLEGO, ALESSANDRO ASCIONE, \\ MARIA LUISA DUPUIS, MARA GELLINI, STEFANO BARCA and MAURIZIO CIANFRIGLIA
}

Section of Pharmacogenetics, Drug Resistance and Experimental Therapeutics, Department of Drug Research and Evaluation, Istituto Superiore di Sanità, Viale Regina Elena 299, I-00161 Rome, Italy

Received September 10, 2007; Accepted November 19, 2007

\begin{abstract}
We report the genetic construction and expression of a fusion protein between a single chain fragment variable (scFv) human antibody (E8) specific for CEA cell surface antigen and yeast cytosine deaminase (yCD). Sequences encoding for the scFvE8 human monoclonal antibody recognizing an epitope shared by CEACAM1, CEACAM3 and CEACAM5 isoforms were assembled with a monomer of yCD. The construct was placed under the transcriptional regulation of the lac promoter, and in frame with 6xHis tag for protein purification. After transformation and induction of E. coli, the protein was recovered from cell lysates and processed for purification. The scFvE8:yCD fusion protein possessed the binding specificity for melanoma (Mel P5) and colon carcinoma (LoVo) cell lines similar to its cognate human scFv antibody. The scFv8:yCD system showed the ability to render tumor cells susceptible to the far less toxic substrate 5-fluorocytosine (5-FC) by its enzymatic conversion into 5-fluorouracil (5-FU). In vitro pre-treatment of Mel P5 and LoVo cell lines with scFvE8:yCD followed by cell washing and incubation with 5-FC, resulted in significant cell killing supporting the utility of this fusion protein as an agent for tumor-selective prodrug activation. This study shows the feasibility of constructing fusion proteins in a prokaryotic cell based system consisting of a human $\mathrm{scFv}$ antibody and $\mathrm{yCD}$ to convert the antifungal agent 5-FC to 5-FU, one of the widely used anticancer agent.
\end{abstract}

Correspondence to: Dr Silvia Zamboni, Section of Pharmacogenetics, Drug Resistance and Experimental Therapeutics, Department of Drug Research and Evaluation, Istituto Superiore di Sanità, Viale Regina Elena 299, I-00161 Rome, Italy

E-mail: silvia.zamboni@iss.it

Abbreviations: 5-FC, 5-fluorocytosine; 5-FU, 5-fluorouracil; $\mathrm{yCD}$, yeast cytosine deaminase; $\mathrm{scFv}$, single chain fragment variable; CEA, carcinoembryonic antigen; GO, glucose oxidase; ADEPT, antibody direct enzyme prodrug therapy

Key words: immunotherapy, single chain fragment variable, carcinoembryonic antigen, cancer, 5-fluorouracil, yeast cytosine deaminase, ADEPT

\section{Introduction}

MAbs directed against tumor-associated antigens have long been the focus of investigation for their potential utility for both the detection and treatment of human malignancies (1). The ability of particular $\mathrm{mAb}$ to specifically localize in tumor tissues in vivo offers a variety of therapeutic approaches for cancer therapy (2). Natural effector functions such as complement activation or antibody-dependent cellular cytotoxicity have been shown to mediate tumor cell destruction in vitro (3), but $\mathrm{mAb}$ alone have not met with consistent clinical success $(4,5)$. Other approaches include chemically conjugating drugs or toxins to $\mathrm{mAb}$ or genetically linking sequences encoding toxins or biologically active domains to $\mathrm{F}\left(\mathrm{ab}^{\prime}\right) \mathrm{s}$ or single chain fragment variable (scFvs) (6). These approaches generally require distribution of the conjugate throughout the tumor mass and efficient internalization of the complex in order to be effective. Each of these requirements has been shown to pose major limitations in the therapy of solid tumors (7).

A promising approach for tumor therapy is to use the antigen binding capability of an antibody to deliver enzymatic activities to tumor tissues, which are then exploited to convert relatively non-toxic prodrugs into more active chemotherapeutic agents. This is a two-step approach in which the antibody-enzyme complex is allowed to localize and clear from the circulation prior to the systemic administration of the prodrug. Thus, the prodrug is converted to an active chemotherapeutic agent by the enzyme which is principally located in tumor tissue. Such an approach may overcome the limitations described above, since the released drug can migrate throughout the tumor mass and exert activity on both the cells that have bound the enzyme conjugate and those that have not. Thus, one should be able to achieve a much higher concentration of drug within tumor masses while minimizing the toxic effects of the drug on non-target tissues. Several studies have now been reported indicating the potential of this two-step approach to cancer therapy (8).

Recently, we described the isolation and the affinitymaturation by mutagenesis in vitro of a new anti-CEA singlechain antibody fragment, scFvE8, from an ETH-2 synthetic antibody library (9). This human antibody recognizes human CEA protein in ELISA, Western blot and flow cytometry. 
Due to its particular capacity to recognize CEACAM1, CEACAM3, and CEACAM5 shared epitope, scFvE8 recognizes a large panel of tumors including melanoma, colon, breast and lung carcinoma. It is particularly interesting that scFvE8 shows no reactivity in various normal human cells, including distinct classes of lymphocyte subpopulations and neutrophils, as well as a broad panel of human organs (9).

The antimetabolite 5-fluorouracil (5-FU) (10) is one of the most active chemotherapeutic agents for the treatment of colorectal cancer, but it has limited efficacy due to gastrointestinal and hematological toxicities (11). High systemic toxicity of 5-FU could be circumvented by introducing gene and/or antibody-directed enzyme prodrug therapy (GDEPT/ADEPT) relying on the ability of bacterial and/or yeast cytosine deaminase (CD) enzyme to convert far less toxic substrate 5-fluorocytosine (5-FC) to 5-FU.

In this study, we report the genetic construction and expression of a fusion protein made of the scFvE8 linked with the $\mathrm{CD}$ from yeast. The fusion scFvE8:yCD protein is produced in Escherichia coli system. It has a specificity that closely mimics the specificity of the parental human antibody scFvE8 and sensitizes CEA-positive cells to the cytosine analog, 5-fluorocytosine (5-FC) due to the enzymatic conversion of 5-FC to the toxic metabolite, 5-FU.

\section{Materials and methods}

Antibodies and reagents. Recombinant human carcinoembryonic antigen (CEA) and recombinant glucose oxidase (GO, EC1.1.3.4) from Aspergillus niger were purchased from Sigma (St. Louis, MO). The scFvE8 anti CEA antibody (9), the scFvGO anti GO antibody (12), and the scFv antiyCD antibody (Mallano, unpublished data) were isolated from the same ETH-2 antibody phage library using an identical biopanning procedure $(13,14) .5$-FC was purchased from Sigma, 5-FU (Mayne Pharma, Naples, Italy) was kindly provided by Dr A. Savarese, IRE, Rome, Italy.

Genetic engineered constructs. The scFvE8 heavy and light chain variable regions were obtained by PCR amplification of pDN332 (9). Oligonucleotide primers used in the PCR were: CEASTU forward primer 5'-CGTTATTAAGGCC TATGGCCGAGGTGCAGCTG-3' contains StuI restriction enzyme sequence and 18-base pair (bp) sequence encoding for the first 7 amino acids of scfvE8. LinCEA reverse primer 5'-TGAGCCGGAAGAGCTACTACCCGATGAGGAA GAGCCTAGGACGGTCAGCTTGGT-3' contains 33 base pair sequences encoding for the first 11 amino acids of linker fragment (SSSSG) ${ }_{3}$, useful for joining yCD enzyme sequence, and the sequence encoding for the last 7 amino acids of scFvE8. The nucleotidic sequence of yCD was amplified by PCR from cDNA inserted in pQE30Xa (Qiagen; Madison, WI) with the primers: LinyCD forward 5'-GGTAGTAGC TCTTCCGGCTCATCGTCCAGCGGCATGGTGACA GGGGGAATGGCAA-3' encoding for the last 11 amino acids of linker sequences and the first 7 amino acid sequence of yCD. The ESyCD reverse primer 5'-ATCCGATATCGTC GACCTCACCAATATCTTC-3' containing SalI restriction enzyme sequence and the last part the sequence encoding for yCD. Both PCR fragments amplified with Pwo enzyme
(Roche Diagnostics, IN, USA) were agarose-purified (High Pure PCR Product Purification Kit, Roche Diagnostics). Portion of the linker present in each PCR product has an overlapping region necessary to assemble chimeric protein by filling reaction (5-min denaturation and 10 cycles $1 \mathrm{~min}$ $94^{\circ} \mathrm{C}, 4 \mathrm{~min} 65^{\circ} \mathrm{C}$ ). Final amplification was carried out with primers $C E A S T U$ and EsyCD (Fig. 1) and the product was agarose-purified, digested with restriction enzymes $S t u \mathrm{I}$ and SalI, and cloned into the plasmid pQE30Xa (Qiagen), containing $6 \mathrm{xHis}$ tag sequence for protein purification. The same procedures were carried out for the construction of an irrelevant fusion protein made by the scFvGO, specific for glucose oxidase (GO) from Aspergillus niger, genetically linked with the gene encoding for $\mathrm{yCD}$ enzyme. All genetic constructs were sequenced by Biofab Research, srl, (Rome, Italy).

Expression and purification. Plasmid pQE30Xa scFvE8:yCD and scFvE8:GO were transformed into the strain of $E$. coli, TG1 [supE hsd $\Delta 5$ thi $\Delta$ (lac-proAB) $F^{\prime}(\operatorname{traD} 36$ proAB+ lacIqlacZ $\Delta \mathrm{M} 15)]$, and the cultures were grown overnight in $2 \mathrm{X}$ TY broth containing $100 \mu \mathrm{g} / \mathrm{ml}^{-1}$ ampicillin and $1 \%$ glucose in a $37^{\circ} \mathrm{C}$ shaker. The culture was diluted $1: 100$ in 1 liter $2 \mathrm{X}$ TY broth lacking exogenous glucose and shaken at $37^{\circ} \mathrm{C}$. When the culture attained $\mathrm{A}_{600}=0.5$, isopropyl- $\beta-\mathrm{D}-$ thiogalactopyranoside (IPTG) (Sigma) was added to a final concentration of $1 \mathrm{mM}$. Cells were harvested $3 \mathrm{~h}$ later. The culture was centrifuged at $10,000 \mathrm{rpm}$ for $20 \mathrm{~min}$ at $4^{\circ} \mathrm{C}$. The fusion proteins were purified by affinity chromatography on Ni-NTA resin (Qiagen), using native protocol according to the manufacturer's instructions (The QIAexpressionist ${ }^{\mathrm{TM}}$ ). Protein concentration was determined with Fernandez-Patron method. yCD enzyme was obtained by induction of expression of the encoding sequence inserted in pQE30Xa vector, in TG1 E. coli. The proteins were purified by affinity chromatography on Ni-NTA resin (Qiagen), as described above.

SDS-PAGE and Western blot analysis. Purified scFvE8:yCD fusion protein was analyzed along with scFvE8 on $10 \%$ SDS-PAGE gel under reducing conditions. Gel was either stained with Fernandez-Patron method or transferred to nitrocellulose membrane in $1 \mathrm{X}$ TG buffer (Bio-Rad, CA, USA) with $20 \%$ methanol, for $90 \mathrm{~min}$ at $140 \mathrm{~V}$. The membrane was blocked in PBS 5\% powdered-milk (M/PBS $5 \%$ ) washed 3 times for $10 \mathrm{~min}$ in PBS and incubated for $2 \mathrm{~h}$ with anti-polyhistidine mouse mAb in 2\% M/PBS (\#H 1029 Sigma-Aldrich, 1:1000), washed 3 times as above, and incubated for $1 \mathrm{~h}$ in polyclonal goat anti-mouse HRP IgG (\#P0447, Dako, Denmark, 1:1000) in M/PBS 2\%. After 3 washings in $2 \% \mathrm{M} / \mathrm{PBS}$, the blot was developed with $\mathrm{DAB}$ buffer, one tablet (10 mg) of 3,3'-diaminobenzidine (Sigma) in $20 \mathrm{ml}$ of PBS and $3 \mu \mathrm{l}$ of hydrogen peroxide $30 \%$, for $3 \mathrm{~min}$. The reaction is stopped with $\mathrm{H}_{2} \mathrm{O}$. ScfvE8:yCD was also detected with the supernatant $\mathrm{scFv}$ specific for $\mathrm{yCD}$ protein, developped in our laboratory. The anti-antiflag Monoclonal antibody M2 (Sigma) was used as secondary antibody at the final concentration of $2 \mu \mathrm{g} / \mathrm{ml}^{-1}$.

ELISA. Ninety-six-well ELISA plates (Nunc, Maxisorp, Denmark) were coated overnight either with $50 \mu 1 /$ well of 
$10 \mu \mathrm{g} / \mathrm{ml}^{-1}$ purified CEA or GO antigen in PBS. Next day a blocking solution of $2 \% \mathrm{M} / \mathrm{PBS}$ was added, and after $2 \mathrm{~h}$ the plates were washed with PBS. Then the plates were incubated for $2 \mathrm{~h}$ at RT with $50 \mu \mathrm{l} /$ well of $10 \mu \mathrm{g} / \mathrm{ml}^{-1}$ of both fusion proteins and $50 \mu \mathrm{l} /$ well of $5 \mu \mathrm{g} / \mathrm{ml}^{-1}$ of scFvE8 or scFvGO. After 3 washes with PBS the wells were incubated at RT with $50 \mu 1$ mixture of antibodies composed of mouse monoclonal anti-polyhistidine antibody (Cat No. H 1029 Sigma, 1:1000) and polyclonal goat anti-mouse HRP IgG (Dako, 1:500) in M/PBS 2\%, for $2 \mathrm{~h}$. After 3 washes the reaction was visualized using 3,31-5,51-tetramethylbenzidine (soluble BM blue POD substrate, Roche Diagnostics) and read at $450 \mathrm{~nm}$ wavelength.

$C E A$ binding specificity of the $s c F v E 8: y C D$. The specific recognition of the cell surface CEA antigen by the scFvE8 and the fusion protein scFvE8:yCD was determined by flow cytometry and Western blot analyses.

In the first study Mel P5 and LoVo cell lines in exponential phase of growth were trypsinized (we verified that CEA antigen is not sensitive to the enzymatic treatment), collected, washed in PBS $1 \%$ BSA and pelletted. Cells $\left(2.5 \times 10^{5}\right)$ were resuspended with $50 \mu 1 \mathrm{PBS}, 1 \% \mathrm{BSA}$ containing $5 \mu \mathrm{g} / \mathrm{ml}^{-1}$ of scFvE8 or scFvE8:yCD primary antibodies and incubated for $1 \mathrm{~h}$ at RT. After several washings, cells were resuspended for $1 \mathrm{~h}$ at $4^{\circ} \mathrm{C}$ in PBS, containing an anti-mouse polyhistidine antibody (SigmaAldrich, 1:1000). At the end of this procedure, the cells were washed and incubated again with $6 \mu \mathrm{g} / \mathrm{ml}^{-1}$ of FITC-labelled goat anti-mouse IgG (\#31541, Pierce, Rockford, IL) for $30 \mathrm{~min}$ at $4^{\circ} \mathrm{C}$. In a parallel experiment, an irrelevant human $\mathrm{scFv}$ antibody directed to glucose oxidase (12) was used as negative control. After staining, the cell samples were washed, maintained at $4^{\circ} \mathrm{C}$ and immediately analyzed by FACScan (Becton-Dickinson and Co., Franklin Lakes, NJ) equipped with $15 \mathrm{nW}$ argon laser. Fluorescence compensation was determined using samples stained with anti-glucose oxidase $\mathrm{scFv}$ and goat FITC-conjugated anti-mouse secondary antibody.

In the second study, Mel P5 and LoVo cell lines were harvested by trypsin (EuroClone, Milan, Italy), washed with cold PBS, resuspended and homogenated in AKT $150 \mathrm{mM} \mathrm{NaCl}$ buffer, $20 \mathrm{mM}$ Tris- $\mathrm{HCl} \mathrm{pH} 7.4,1 \%$ NP40, $10 \%$ glycerol, in presence of inhibitors proteases (\#P8849, Sigma Aldrich). After centrifugation at $13000 \mathrm{rpm}$ for $15 \mathrm{~min}$, the supernatant was harvested and the protein total concentrations extimated with Bradford assay. Total proteins $(240 \mu \mathrm{g})$ were fractionated on $6.5 \%$ SDS-PAGE under reducing conditions, transferred onto a nitrocellulose membrane and blocked in 5\% M/PBS. After washing in PBS with $0.05 \%$ Tween-20 (T/PBS), the membrane was incubated with $10 \mu \mathrm{g} / \mathrm{ml}^{-1}$ of scFvE8 or scFvE8:yCD fusion protein in $2 \% \mathrm{M} / \mathrm{PBS}$ for $1 \mathrm{~h}$, washed in T/PBS, and incubed for $1 \mathrm{~h}$ with the mouse $\mathrm{mAb}$ anti-polyhistidine antibody (Sigma-Aldrich) in M/PBS 2\%. After washing in T/PBS, specific binding of scFvE8 or scFvE8:yCD was revealed with anti-mouse HRP IgG (Dako, 1:500) using ECL kit (Pierce, Rockford, IL).

Functional assays of the scFvE8:yCD on cells. The ability of the scFvE8:yCD fusion protein to convert far less toxic substrate 5-fluorocytosine (5-FC) to 5-FU was tested in two different investigations using a cell-based system. The cells used are Mel P5 derived from a human primary melanoma (15) and the human colon adenocarcinoma LoVo cells. Both cell lines were maintained in a basic medium (BM) constituted by RPMI-1640 (EuroClone) supplemented with $10 \%$ fetal bovine serum (EuroClone) and $1 \%$ penicillinstreptomycin in humidified atmosphere with $5 \% \mathrm{CO}_{2}$ at $37^{\circ} \mathrm{C}$.

In the first assay, Mel P5 and LoVo cells were seeded into 96-well microtiter plates (Corning Cable Systems srl, Turin, Italy) at 2500 cells/well in BM containing $3 \mu \mathrm{g} / \mathrm{ml}^{-1}$ of scFvE8:yCD and different concentrations of 5-FC. The plates were incubated at $37^{\circ} \mathrm{C}$ for 4 days and cell viability extimated by WST-1 assay (Takara, VinciBiochem, Vinci, Florence, Italy). In the second assay Mel P5 and LoVo cells were seeded as above and allowed to adhere overnight. Then, medium was removed and a fresh BM containing $10 \mu \mathrm{g} / \mathrm{ml}^{-1}$ of scFvE8:yCD was added. After 4-h incubation, the medum was changed and a fresh BM containing different concentrations of 5-FC, was added. Cell viability was determined after 4-day culture using WST-1 Takara assay.

Different concentrations of 5-FC and 5-FU alone were used respectively as positive and negative controls of cell vitality. yCD enzyme alone was not as cytotoxic as scFvE8:yCD alone (data not shown). Results are the mean of triplicate samples.

\section{Results}

Genetic engineering of the scFv antibodies for ADEPT. To drive the enzymatic activity of yCD to CEA-expressing tumor cells a fusion protein was genetically engineered using the cDNA derived from CEA-specific antibody scFvE8 previously described (9). As control for ADEPT studies an irrelevant fusion protein composed by glucose oxidase (GO) specific scFv antibody (12), and yCD was genetically constructed. Antigen GO from Aspergillus niger is not present in mammalian cells. The cDNA corresponding to the open reading frame of yCD (16) was appended to the $3^{\prime}$ end of the cDNA encoding for the antibodies scFvE8 or scFvGO by a linker of $45 \mathrm{bp}(\mathrm{SSSSG})_{3}$. The cDNA constructs were cloned into the pQE30Xa (Qiagen), containing 6xHis tag sequence for protein purification (Fig. 1A). PCR bands of the genetic constructs corresponding to $1300 \mathrm{bp}$, obtained after fill-in procedures are shown in Fig. 1B. The clones isolated after trasformation of TG1 E. coli bacterial strain, were characterized under genetic-molecular aspect. The complete amino acid sequence of the genetic constructs scFvE8:yCD and scFvGO:yCD were shown in Fig. 2.

The fusion proteins scFvE8:yCD and scFvGO:yCD were purified from the pellet of TG1 strain $E$. coli by affinity chromatography. The yield was about $150 \mu \mathrm{g} / \mathrm{ml}^{-1}$ for each fusion protein. The expected 45 to $50-\mathrm{kDa}$ size of the scFvE8:yCD and scFvGO:yCD constructs was confirmed by Western blot studies using an anti-polyhistidine antibody to detect the fusion proteins after SDS-PAGE migration (Fig. 1C).

In order to assess the exact expression of the $\mathrm{yCD}$ moiety of the scFvE8:yCD construct, the fusion protein 


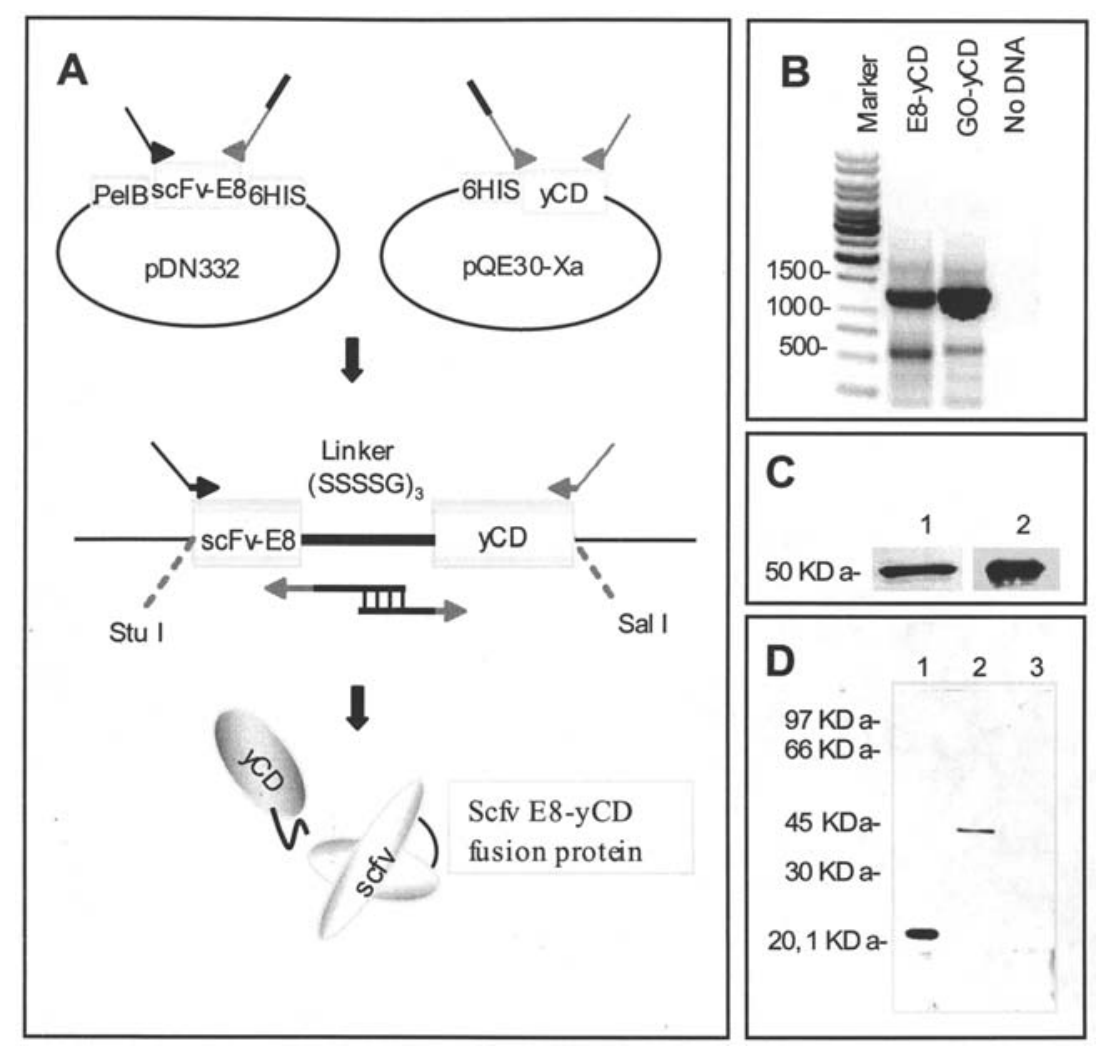

Figure 1. Schematic representation of the engineered scFvE8:yCD fusion protein. (A) The genes encoding for the human scFv antibody to CEA (E8) and cytosine deaminase from yeast (yCD) were assembled by a (SSSSG) 3 linker, inserted in the prokaryotic vector pQE30Xa and expressed in TG1 strain of $E$. coli to form a bifunctional protein. (B) The PCR-DNA fragments corresponding to the expected 1250 bases pair encoding for scFvE8:yCD and the irrelevant fusion protein scFvGO:yCD are shown. (C) The result of Western blot analysis of the purified fusion proteins scFvE8:yCD (lane 1) and scFvGO:yCD (lane 2). The $50 \mathrm{kDa}$-His tagged fusion proteins were detected with an anti-polyhistidine secondary antibody as described in the text. (D) The scFvE8.yCD protein was detected by Western blot as a band of approximately $50 \mathrm{kDa}$ with an anti-yCD antibody (in scFv format).

SCFVGO:YCD Amino acid sequence

MAEVQLVESGGGLVQPGGSLRLSCAASGFTFSSYAMSWVRQAPGK GLEWVSAISGSGGSTYYADSVKGRFTISRDNSKNTLYLQMNSLRA EDTAVYYCAKWNNWRNFDYWGQGTLVTVSRGGGGSGGGGSGGGGS SELTQDPAVSVALGQTVRITCQGDSLRSYYASWYQQKPGQAPVLV IYGKNNRPSGIPDRFSGSSSGNTASLTITGAQAEDEADYYCNSSE LPPVYVVFGGGTKLTVLGSSSSGSSSSGSSSSGVTGGMASKWDQK GMDIAYEEAALGYKEGGVPIGGCLINNKDGSVLGRGHNMRFQKGS ATLHGEISTLENCGRLEGKVYKDTTLYTTLSPCDMCTGAIIMYGI PRCVVGENVNFKSKGEKYLQTRGHEVVVVDDERCKKIMKQFIDER PQDWFEDIGE

SCFVE8:YCD Amino acid sequence

MAEVQLAESGGGLVQPGGSLRLSCAASGFTFSSDAMSWVRQAPGK GLEWVSAISGSGGSTYYADSVKGRFTISRDNSKNTLYLQMNSLRA EDTAVYYCAKSNEFLFDYWGQGTLVTVSRGGGGSGGGGSGGGGSS ELTQDPAVSVALGQTVRITCQGDSLRSSYASWYRQRPGQAPVPVI YGKNNWPSGIPDRFSGSSSGNTASLTITGAQAEDEADYYWNSSYA WLPYVVFGGGTKLTVLGSSSSGSSSSGSSSSGVTGGMASKWDQKG MDIAYEEAALGYKEGGVPIGGCLINNKDGSVLGRGHNMRFOKGSA TLHGEISTLENCGRLEGKVYKDTTLYTTLSPCDMCTGAIIMYGIP RCVVGENVNFKSKGEKYLQTRGHEVVVVDDERCKKIMKQFIDERP QDWFEDIGE

Figure 2. Amino acid sequences. The complete amino acid sequences in a single letter code of the scFvE8:yCD and the scFvGO:yCD fusion proteins are shown. CDR1, CDR2, CDR3 are underlined.

was biochemically investigated by an antibody specific for $\mathrm{yCD}$ in scFv format. As shown in Fig. 1D, the anti-yCD antibody, reacts with yCD (lane 1) and scFvE8:yCD (lane 2) at the expected migration in SDS-PAGE and corresponds to $18-20 \mathrm{kDa}(\mathrm{MW}$ of $\mathrm{yCD}$ ) or $45-50 \mathrm{kDa}$ (MW of scFvE8:yCD). The same antibody does not react with GO irrelevant antigen (lane 3).

The antigen specificity of the engineered fusion proteins. Flow cytometry, immunobiochemical and ELISA investigations were performed in order to assess the specific antigen recognition of the engineered scFvE8:yCD. When tested in flow cytometry the scFvE8:yCD showed weaker binding profiles on Mel P5 and LoVo cells in comparison with the original scFvE8 antibody (Fig. 3A). This difference in the antigen recognition may be due to an alterated His-tag exposure in the fusion protein. To this regard, we observed a lower binding level of the original scFvE8 on CEAexpressing cells when the scFvE8 reactivity was detected with an anti-polyhistidine secondary antibody in comparison with an anti-FLAG-tag secondary antibody (data not shown).

Further, ELISA studies summarized in Table I demonstrate that the specific binding activity of the scFvE8:yCD is retained to a degree comparable to the parental scFvE8 and that the DNA recombinant procedures utilized it gave effective fusion protein. In fact, scFvE8:yCD was very effective in the recognition of CEA specific antigen either in cellular total extract or in the purified recombinant protein (Fig. 3B). It is noteworthy that the high staining of Mel P5 


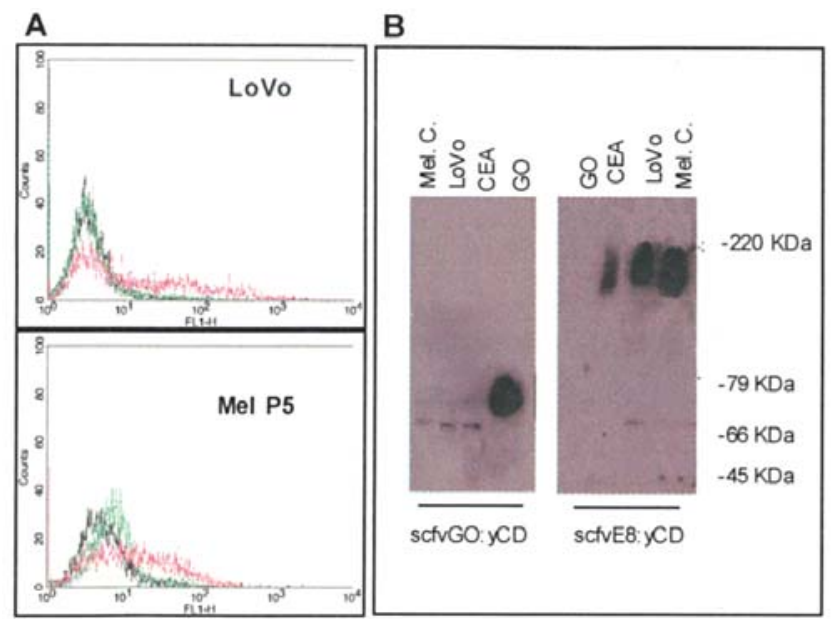

Figure 3. Specificity of the scFvE8:yCD fusion protein. (A) The flow cytometry profiles representing the binding level of the scFvE8:yCD (red line), the irrelevant scFvGO:yCD (black line) and the parental scFvE8 (green line) on living intact/LoVo and Mel P5 cell lines are shown. (B) Total extracts from LoVo and Mel P5 cell lines were run on SDS-PAGE in parallel with CEA and GO. After incubation with scFvE8:yCD or scFvGO:yCD the specific reactivity was revealed by an anti-polyhistidine secondary antibody. Both fusion proteins retain the binding specificity for respective antigens.

Table I. Specificity of the scFv antibodies and fusion proteins by ELISA.

\begin{tabular}{lcccc}
\hline scFv antibodies $^{\mathrm{a}}$ & \multicolumn{5}{c}{ Antigens $^{\mathrm{b}}$} \\
\hline & CEA & GO & yCD & scFvE8:yCD \\
\hline scFvE8 & $+++^{\mathrm{c}}$ & - & ND & ND \\
scFvE8:yCD & ++ & - & ND & ND \\
scFvGO & - & +++ & ND & ND \\
scFvGO:yCD & - & +++ & ND & ND \\
scFv anti-yCD & ND & ND & +++ & ++ \\
\hline
\end{tabular}

${ }^{a}$ The $\mathrm{scFv}$ antibodies isolated by bio-panning and derivated fusion proteins are the following: scFvE8 and scFvE8:yCD are specific for CEA; scFvGO and scFvGO:yCD are specific for GO; scFvyCD, is specific for $\mathrm{yCD}$. ${ }^{\mathrm{b}} \mathrm{CEA}$ and $\mathrm{GO}$ are purified proteins; $\mathrm{yCD}$ is expressed and purified as described in the text. 'ELISA signals are indicated: -, for equal or low background; ++ or +++, more than three and four times the background, respectively. ND, not determined.

lysates in Western blot corresponds with the high reactivity of the scFvE8 antibody on melanoma cells (9).

Functional assay of the scFvE8:yCD. ScFvE8:yCD fusion protein was evaluated for the ability to control tumor cell growth of CEA-expressing cells by converting the antifungal agent 5-FC to the highly toxic 5-FU. Cell sensitivity to 5-FC, 5-FU, and selective cytotoxic effect mediated by ScFvE8:yCD in the presence of 5-FC was assessed on tumor cells Mel P5 and LoVo in vitro. These cell lines were
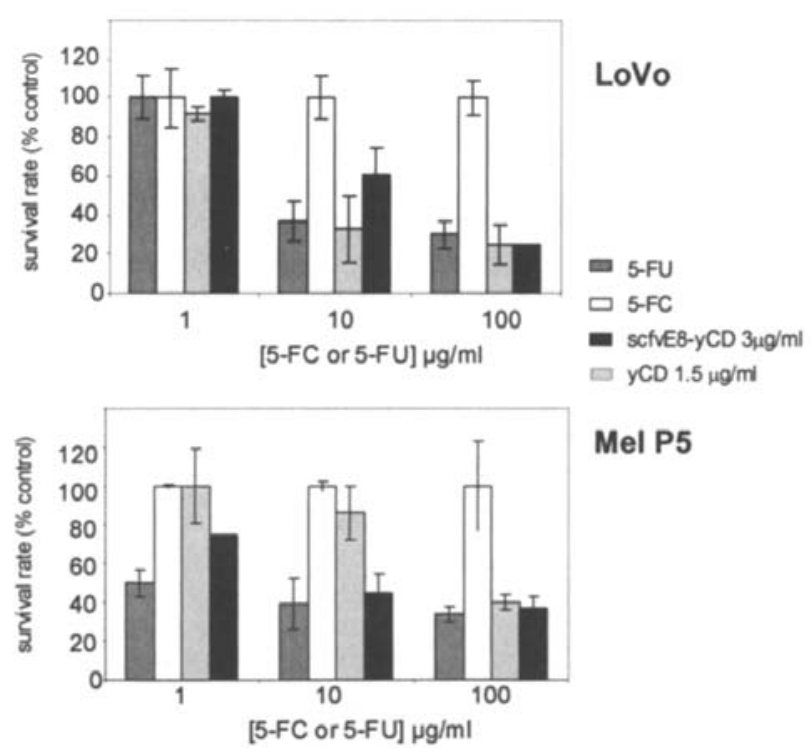

Figure 4. Functional assay of the scFvE8:yCD fusion protein. Cell sensitivity to 5-FC, 5-FU, and the selective cytotoxic effect mediated by ScFvE8:yCD was assessed on tumor cells Mel P5 and LoVo. Antifungal agent 5-FC was converted to the highly toxic 5-FU by scFvE8:yCD or free $\mathrm{yCD}$. The cell lines were seeded (2500 cell/well) and cultured in BM for 4 days containing either $3 \mu \mathrm{g} / \mathrm{ml}^{-1}$ of scFvE8:yCD or $1.5 \mu \mathrm{g} / \mathrm{ml}^{-1}$ of yCD (identical molar concentration for free $\mathrm{yCD}$ and $\mathrm{yCD}$ fused with $\mathrm{scFvE}$ ) in presence of the indicated concentrations of 5-FC. Cell cytotoxicity was evaluated by WST-1 assay and calculated as a percentage of survived cells. Values are reported as the mean of triplicate samples. The bars indicate SD.

cultured in BM for 4 days containing either $3 \mu \mathrm{g} / \mathrm{ml}^{-1}$ of scFvE8:yCD or $1.5 \mu \mathrm{g} / \mathrm{ml}^{-1}$ of yCD and different concentrations of 5-FC (Fig. 4). Cell cytotoxicity evaluated by WST-1 assay shows that scFvE8:yCD and yCD exert a similar cell growth inhibition in the presence of $100 \mu \mathrm{g} / \mathrm{ml}^{-1}$ of 5-FC while no effect was observed either in presence of 5-FC $\left(100 \mu \mathrm{g} / \mathrm{ml}^{-1}\right)$ or scFvE8:yCD fusion protein or yCD alone. When LoVo and Mel P5 cell lines, were preincubated with scFvE8:yCD at $10 \mu \mathrm{g} / \mathrm{ml}^{-1}$ for $4 \mathrm{~h}$, washed and then cultured for 4 days with $\mathrm{BM}$ containing different concentrations of 5-FC, we observed that pre-treatment with scFvE8-yCD significantly inhibited cell growth in comparison with the same treatment in the presence of irrelevant scFvGO:yCD fusion protein (Fig. 5).

The above observation it is of particular interest since the growth inhibition exerted by scFvE8-yCD in the presence of 5-FC depends on the specific binding of the fusion protein on CEA-expressing cells and the subsequent conversion of 5-FC to 5-FU at the tumor cell level.

\section{Discussion}

Previous studies have demonstrated that significant in vitro and in vivo activities can be obtained using $\mathrm{mAb}-\mathrm{yCD}$ conjugates to convert 5-FC into the antitumor agent 5-FU $(17,18)$. In several studies mAb-enzyme fusion protein was prepared using chemical cross-linking reagents that react with amino acid side chains on each individual protein of the conjugates (8). In general, the utilization of chemical procedures that has been largely applied for ADEPT resulted in reagents having an inherent lack of specificity. The 

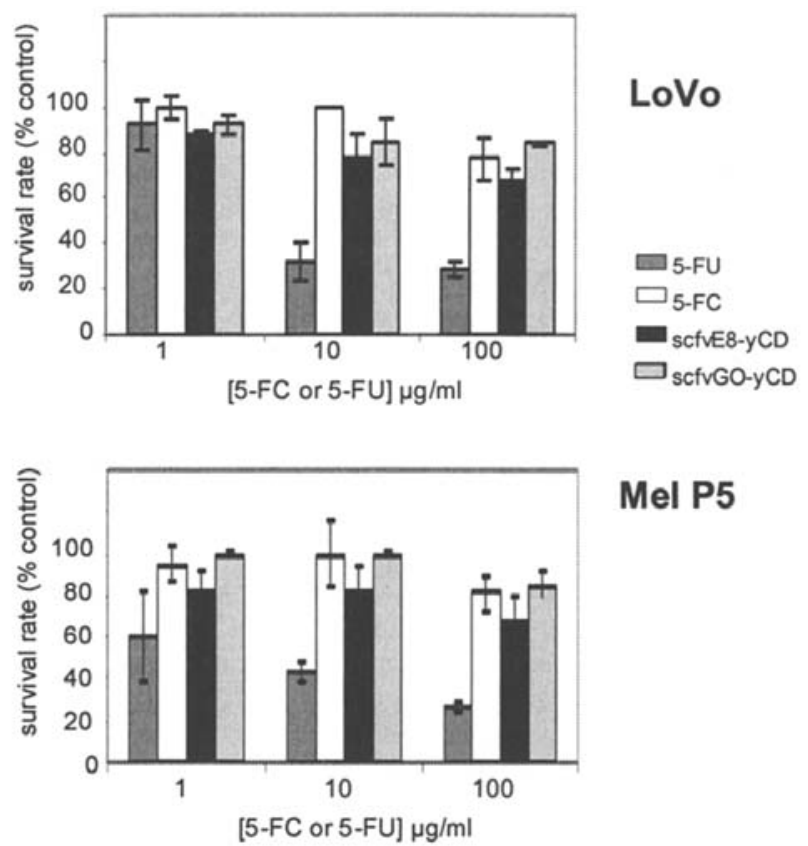

\section{Mel P5}

Figure 5. Cell specificity of the 5-FC activation driven by the antibody of fusion protein. The in vitro cytotoxic effect of the prodrug 5-FC on Mel P5 and LoVo cells was evaluated by pre-incubating the cells (2000 cells/well) with either scFvE8:yCD or scFvGO:yCD for $4 \mathrm{~h}$. After washing the cells were cultured for 4 days in BM and different concentration of 5-FC. Antibody binding dependent cell cytotoxicity, was evaluated by WST-1 assay and calculated as a percentage of survived cells. Values are reported as the mean of triplicate samples. The bars indicate SD

resulting conjugates are composed of highly complex mixture with various degrees of binding and enzymatic activities (8). Differently, recombinant DNA technologies offer the opportunity to design and produce well defined molecules with bifunctional activities $(4,19)$. Human monoclonal scFvs derived from phage display antibody library (20) afford the opportunity of developing recombinant molecules maintaining the antigen-binding characteristics of the $\mathrm{scFv}$ parental antibody together with the competence of the desired enzyme (21). Such scFv antibodies have been shown to offer advantages over whole antibody with respect to tumor penetration and clearance from the circulation (19). Furthermore, it is possible to fuse genetically such scFv to sequences encoding other functional domains, providing molecules with multiple activities with minimal size $(22,23)$. Another advantage of genetically engineering scFv molecules of minimal complexity is that such fusion proteins can be produced using prokaryotic expression systems which grow rapidly and inexpensively (19).

Recombinant fusion protein for ADEPT based on the humanization of rabbit gene encoding for $\mathrm{scFv}$ but retaining the rodent CDRs (24) and catalytic activity of the monomer of yCD has been already described (25). In the present study, we show that a fully human $\mathrm{scFv}(\mathrm{scFvE8})$ to CEA was genetically combined with yCD, expressed in E. coli system and readily purified in native conditions by cell lysates; once recovered the fusion protein maintains the functional activities of both the respective parental molecules.

Fig. 3 and Table I show that CEA is identically recognized by scFvE8 and scFvE8:yCD both in ELISA and Western blot analysis. In contrast, flow cytometry investigation shows a decrease in the binding level of scFvE8:yCD in comparison with scFvE8. This difference in the CEA recognition on the cell surface of living/intact cells may be due for technical reasons. These may include a different His tag exposure in chimeric proteins in comparison with $\mathrm{scFv}$.

Nonetheless, the data reported in Fig. 3 and in Table I demonstrate that the parental and the conjugated scFvE8 to CEA possess similar specificity. Thus, none of the genetic manipulation appears to have impacted its target antigen specificity.

We demonstrated that the simultaneous presence of scFvE8:yCD and 5-FC generated a toxic effect, resulting in increase in the 5-FC sensitivity of human cancer Mel P5 and LoVo cells in vitro compared with the incubation of scFvE8yCD and 5-FC alone. In addition we observed that fusion protein maintained similar toxicity of $\mathrm{YCD}$ in the presence of 5-FC. Further, pre-incubation of Mel P5 and LoVo cells with scFvE8:yCD followed by cell washing and 4-day exposure to 5-FC resulted in a cell growth inhibition when compared with the same treatment in the presence of irrelevant scFvGO:yCD fusion protein (Fig. 5).

The yCD monomer of the fusion scFvE8:yCD is correctly expressed in the E. coli system since the specific monoclonal antibody to yCD recognizes the enzyme both in Western blotting and ELISA studies (Mallano, unpublished data).

When scFvE8:yCD fusion protein showed effective dual function, either its monomer has catalytic activity, or it can form dimers in solution or after antigen binding. While the homoexameric and dimeric structures of $\mathrm{CD}$ from bacteria and yeast respectively, supports monomer activity (26), both above hypotheses would explain the lower catalytic activity of scFvE8:yCD alone. The data here reported and discussed indicate that this new scFvE8:yCD fusion protein meets several criteria for a potential anticancer compound: i) the $\mathrm{scFv}$ antibody is fully human thus substantially reducing the immunogenicity of the dual construct; ii) the low molecular weight $(50 \mathrm{kDa})$ of the scFvE8:yCD may have favourable diffusion characteristics in solid tumors (7); and iii) it binds selectively and with good affinity to a CEA epitope shared by CEACAM1, CEACAM3 and CEACAM5 isoforms which is expressed on several malignancies including melanoma (27). This CEA epitope is a particularly attractive target for immunotherapeutic purposes because of its expression profile in solid tumor. In fact this epitope has low or absent expression in normal adult tissues and in various normal human cells, including distinct classes of lymphocyte subpopulations and neutrophils (9).

In conclusion, we have described a novel fusion protein, scFvE8:yCD, based on the human scFvE8 to CEA and yCD enzyme relying on the ability to convert far less toxic substrate 5-FC (currently administrates during chemotherapy to combat opportunistic infections) to 5-FU (28). Future studies will determine the ability of this molecule to localize in tumors and activate prodrug in vivo.

\section{Acknowlegements}

This work was in part supported by ISS-NIH research project and by Finalized Research of the Italian Ministry of Health. 


\section{References}

1. Adams GP and Weiner LM: Monoclonal antibody therapy of cancer. Nat Biotechnol 23: 1147-1157, 2005.

2. Beckman RA, Weiner LM and Davis HM: Antibody constructs in cancer therapy: protein engineering strategies to improve exposure in solid tumors. Cancer 109: 170-179, 2006.

3. Carter PJ: Improving the efficacy of antibody-based cancer therapies. Nat Rev Cancer 1: 118-129, 2001.

4. Glennie MJ and van de Winkel JG: Renaissance of cancer therapeutic antibodies. Drug Discov Today 8: 503-510, 2003.

5. Waldmann TA: Immunotherapy: past, present and future. Nat Med 9: 269-277, 2003.

6. Sharkey RM and Goldenberg DM: Targeted therapy of cancer: new prospects for antibodies and immunoconjugates. CA Cancer J Clin 56: 226-243, 2006.

7. Christiansen J and Rajasekaran KA: Biological impediments to monoclonal antibody-based cancer immunotherapy. Mol Cancer Ther 3: 1493-1501, 2004.

8. Bagshawe KD: Antibody-directed enzyme prodrug therapy (ADEPT) for cancer. Expert Rev Anticancer Ther 6: 1421-1431, 2006.

9. Pavoni E, Flego M, Dupuis ML, Barca S, Petronzelli F, Inastasi AM, D'Alessio V, Pelliccia A, Vaccaio P, Monteriu G, Ascione A, De Santis R, Felici F, Cianfriglia M and Minenkova O: Selection, affinity maturation and characterization of a human $\mathrm{scFv}$ antibody against CEA protein. BMC Cancer 6: 41-56, 2006.

10. Heidelberger C, Kaldor G, Mukherjee KL and Danneberg PB: Studies on fluorinated pyrimidines. XI. In vitro studies on tumor resistance. Cancer Res 20: 903-909, 1960.

11. Douillard JY, Cunningham D, Roth AD, Navarro M, James RD, Karasek P, Jandik P, Iveson T, Carmichael J, Alakl M, Gruia G, Awad L and Rougier P: Irinotecan combined with fluorouracil compared with fluorouracil alone as first-line treatment for metastatic colorectalcancer: a multicentre randomised trial. Lancet 355: 1041-1047, 2002.

12. Ascione A, Flego M, Zamboni S, De Cinti E, Dupuis ML and Cianfruglia $\mathrm{M}$ : Isolation and characterization of the human monoclonal antibodies $\mathrm{C} 10$ in single chain fragment variable ( $\mathrm{scFv}$ ) format to glucose oxidase from Aspergillus niger. Hybrid Hybridomics 23: 380-384, 2004.

13. Pini A, Viti F, Santucci A, Carnemolla B, Zardi L, Neri P and Neri D: Design and use of a phage display library. Human antibodies with subnanomolar affinity against a marker of angiogenesis eluted from a two-dimensional gel. J Biol Chem 273: 21769-21776, 1998.

14. Silacci M, Brack S, Schirru G, Marlind J, Ettorre A, Merlo A, Viti F and Neri D: Design, construction and characterization of a large synthetic human antibody phage display library. Proteomics 5: 2340-2350, 2005.

15. Luciani F, Spada M, De Milito A, Molinari A, Rivoltini L, Montanaro A, Marra M, Lugini L, Logozzi M, Lozupone F, Federici C, Iessi E, Parmiani G, Arancia G, Belardelli F and Fais $S$ : Effect of proton pump inhibitor pretreatment on resistance of solid tumors to cytotoxic drugs. J Natl Cancer Inst 22: 1702-1713, 2004.
16. Erbs P, Exinger F and Jund R: Characterization of the Saccharomyces cerevisiae FCY1 gene encoding cytosine deaminase and its homologue FCA1 of Candida albicans. Curr Genet 31: 1-6, 1997

17. Aboagye EO, Artemov D, Senter PD and Bhujwalla ZM: Intratumoral conversion of 5-fluorocytosine to 5-fluorouracil by monoclonal antibody-cytosine deaminase conjugates: noninvasive detection of prodrug activation by magnetic resonance spectroscopy and spectroscopic imaging. Cancer Res 58: 4075-4078, 1998.

18. Wallace PM, MacMaster JF, Smith VF, Kerr DE, Senter PD and Cosand WL: Intratumoral generation of 5-fluorouracil mediated by an antibody-cytosine deaminase conjugate in combination with 5-fluorocytosine. Cancer Res 54: 2719-2723, 1994.

19. Holliger P and Hudson PJ: Engineered antibody fragments and the rise of single domains. Nat Biotechnol 22: 1126-1136, 2005.

20. Hoogenboom HR: Selecting and screening recombinant antibody libraries. Nat Biotechonol 23: 1105-1116, 2005.

21. Carter PJ: Potent antibody therapeutics by design. Nat Rev Immunol 6: 343-357, 2006.

22. Halin C, Niesner U, Villani ME, Zardi L and Neri D: Tumortargeting properties of antibody-vascular endothelial growth factor fusion proteins. Int J Cancer 102: 109-116, 2002.

23. Ebbinghaus C, Ronca R, Kaspar M, Grabulovski D, Berndt A, Kosmehl H, Zardi L and Neri D: Engineered vascular-targeting antibody-interferon-gamma fusion protein for cancer therapy. Int J Cancer 116: 304-313, 2005.

24. Rader C, Ritter G, Nathan S, Elia M, Gout I, Jungbluth AA, Cohen LS, Welt S, Old LJ, and Barbas CFIII: The rabbit antibody repertoire as a novel source for the generation of therapeutic human antibodies. J Biol Chem 275: 13668-13676, 2000.

25. Deckert PM, Renner C, Cohen LS, Jungbluth A, Ritter G, Bertino JR, Old L and Welt S: A33scFv-cytosine deaminase: a recombinant protein construct for antibody-directed enzymeprodrug therapy. Br J Cancer 88: 937-939, 2003.

26. Ireton GC, McDermott G, Black ME and Stoddard BL: The structure of Escherichia coli cytosine deaminase. J Mol Biol 315: 687-697, 2002 .

27. Ebrahimnejad A, Streichert T, Nollau P, Horst AK, Wagener C, Bamberger AM and Brummer J: CEACAM1 enhances invasion and migration of melanocytic and melanoma cells. Am J Pathol 165: 1781-1787, 2004.

28. Schiel X, Link H, Maschmeyer G, Glass B, Cornely OA, Buchheidt D, Wilhelm M, Silling G, Helmerking M, Hiddemann W, Ostermann $\mathrm{H}$ and Hentrich $\mathrm{M}$ : A prospective, randomized multicenter trial of the empirical addition of antifungal therapy for febrile neutropenic cancer patients: results of the Paul Ehrlich Society for Chemotherapy (PEG) Multicenter Trial II. Infection 34: 118-126, 2006. 